\title{
Tear Meniscus, Corneal Topographic and Aberrometric Changes After Botulinum Toxin-A Injection in Patients With Blepharospasm and Hemifacial Spasm
}

Neslihan Bayraktar Bilen ( $\nabla$ dr.neslibayraktar@gmail.com )

Ankara Bilkent City Hospital https://orcid.org/0000-0002-6600-6822

Şule Bilen

Ankara City Hospital: Ankara Sehir Hastanesi

Pınar Topçu Yılmaz

Private Practice

Özlem Evren Kemer

Ankara City Hospital: Ankara Sehir Hastanesi

\section{Research Article}

Keywords: blepharospasm, botulinum neurotoxin-A, corneal topography, hemifacial spasm, tear meniscus.

Posted Date: April 19th, 2021

DOI: https://doi.org/10.21203/rs.3.rs-222670/v1

License: (c) (i) This work is licensed under a Creative Commons Attribution 4.0 International License.

Read Full License 


\section{Abstract}

Purpose: To investigate the effect of Botulinum neurotoxin-A (BTX-A) treatment on dry eye symptoms, tear meniscus, corneal topography and corneal aberrometry in patients with benign essential blepharospasm (BEB) and hemifacial spasm (HFS).

Materials and Methods: This prospective study comprised of 32 eyes from 8 BEB and 24 HFS patients. Tear meniscus height (TMH) and depth (TMD), tear break-up time (TBUT), corneal fluorescein staining score (CFSS), Schirmer I test, ocular surface disease index (OSDI) score, corneal topography [corneal power of flat axis (K1), corneal power of steep axis (K2), mean corneal power (Km), astigmatism and thinnest pachymetry] and anterior corneal aberrometry [spherical aberration (SA), vertical coma (vcoma), horizontal coma (hcoma), higher order root mean square (hRMS) and total RMS] were evaluated before BTX-A treatment and 3 weeks after BTX-A treatment.

Results: 3 weeks after BTX-A injection TMH $(265.9 \pm 116.4 \mu \mathrm{m}$ vs. $347.1 \pm 172.6 \mu \mathrm{m} ; p<0.001)$ and TMD $(178.1 \pm 57.7 \mu \mathrm{m}$ vs. $221.4 \pm 90.3 \mu \mathrm{m} ; p<0.001)$ showed a significant increase. TBUT, CFSS, Schirmer I test values were similar $(p>0.005)$. OSDI scores decreased significantly from $27.8 \pm 26.1$ to $18.4 \pm 19.8$ $(p=0.04) . \mathrm{K} 2(44.1 \pm 1.7 \mathrm{D}$ vs. $43.9 \pm 1.7 \mathrm{D} p=0.009), \mathrm{Km}(43.7 \pm 1.6 \mathrm{D}$ vs. $43.6 \pm 1.6 \mathrm{D} p=0.025)$ and astigmatism ( $0.9 \pm 0.6 \mathrm{D}$ vs. $0.7 \pm 0.5 \mathrm{D} ; p=0.02)$ values improved significantly at 3 weeks. Pachymetry and aberrometric values did not change significantly.

Conclusion: BTX-A injection increases tear meniscus and decrease symptoms related to dry eye disease in BEB and HFS patients. It decrease astigmatism and keratometry values but it does not cause a significant change in corneal aberrations.

\section{Introduction}

Essential blepharospasm (BEB) and hemifacial spasm (HFS) are two facial movement disorders characterized by involuntary and frequent contraction of the orbicularis oculi muscle which causes functional disability $[1,2]$. Botulinum neurotoxin-A (BTX-A) injection is the gold standard treatment of these two diseases. It has an anticholinergic effect at the neuromuscular junction by inhibiting acetylcholine release and signal transduction. As the effect is temporary and lasts 3-6 months, repeated injections are required [3].

The frequent and strong closure of eyelids in BEB and HFS can affect tear production, tear distribution, tear drainage and cause dry eye disease [4, 5]. Also, BTX-A treatment was reported as a cause of dry eye disease in the Tear Film and Ocular Surface Society's Dry Eye Workshop (TFOS DEWS) II Report [6]. Ho and colleagues discerned a significant decrease in TBUT and Schirmer test results after BTX-A injections. When the injection side is in the lateral part of the orbicularis oculi muscle, BTX-A diffuses through the orbital septa to the lacrimal gland and decreases tear production via an anticholinergic effect on the lacrimal gland [7]. Also lagophthalmos, incomplete blinking and ectropion are other causes of dry eye associated with BTX-A treatment [8]. However, Gunes et al. have shown that BTX-A treatment significantly 
improved TBUT, OSDI score and CFSS in facial dystonias [9]. The effect of BTX-A on dry eye disease is controversial.

The tear film and ocular surface are affected in dry eye disease (DED); as tear film thickness changes with each blink, tear meniscus measurement is used to show total tear volume [10]. The anterior segment optical coherence tomography (AS-OCT) provides high resolution images of the cornea and anterior segment and can be used for the non-invasive measurement of tear meniscus [11]. Lower tear meniscus measurements correlate well with symptoms and signs of DED $[12,13]$. There is only one study evaluating the effect of BTX-A on the tear meniscus using AS-OCT [4].

Involuntary eyelid spasms cause abnormal pressure on the corneal surface, leading to changes in corneal topography, especially in astigmatism $[9,14]$. DED itself increases irregular astigmatism and higher order aberrations (HOA) [15]. Yakibu and Yoshihiko et al. reported ocular wavefront changes after BTX-A treatment in patients with BEB and HFS $[16,17]$. Also it is reported corneal HOA was correlated with subjective patient reported symptoms in subjects with dry eye [18]. However, to date, there have been no studies evaluating corneal aberrometric changes after BTX-A injections in patients with BEB and HFS.

The aim of this study was to investigate the effect of BTX-A injections on dry eye signs and symptoms and corneal topographic and aberrometric parameters in patients with BEB and HFS as measured before the treatment and 3 weeks after the treatment.

\section{Materials And Methods}

Eight patients with BEB and twenty four patients with HFS involving the eyelids were included in this prospective study. Subjects who had a history of ocular trauma, previous ocular surgery or an ocular surface disease other than dry eye, used contact lenses or topical drops, had a refractive error greater than $\pm 4 \mathrm{D}$ or a history of neurological disorder other than dystonia were excluded from the study. The study protocol was approved by the local ethics committee and adhered to the tenets of the Declaration of Helsinki. Informed consent was obtained from all participants prior to enrollment.

BTX-A (Botox ${ }^{\circledR}$ ) was diluted with $2 \mathrm{ml}$ of $0.9 \%$ sodium chloride in order to obtain $5 \mathrm{U} / 0.1 \mathrm{ml}$ injection. The toxin was injected subcutaneously with a 27-gauge insulin syringe in 5 to 6 selected sites, such as the orbicularis oculi muscle pretarsal fibers, procerus, frontalis muscle and corrugator. The total injected dose was $50-70$ units for each patient.

Patients were evaluated in terms of tear function and corneal topographic parameters before BTX-A treatment and 3 weeks after the injection.

Tear function was evaluated with measurements of lower tear meniscus height (TMH), tear meniscus depth (TMD), tear break-up time (TBUT), corneal fluorescein staining score (CFSS) (National Eye Institute grading scale) and Schirmer I test with anesthesia. TMH and TMD measurements were performed using Swept Source OCT (SS-OCT, DRI OCT Triton, Topcon, Tokyo, Japan). SS AS-OCT single vertical scan 
mode was used to measure TMH and TMD. After measurements were performed, a built-in caliper was used to measure the meniscus distance in micrometers between the cornea (superiorly) and the eyelid (inferiorly) (TMH) and the line from the TMH to inferior fornix (TMD).

Corneal topography measurement was performed by Pentacam (Oculus, Wetzlar, Germany). The corneal power of flat axis (K1), corneal power of steep axis (K2), mean corneal power (Km), corneal astigmatism, thinnest corneal thickness and anterior corneal aberrations (spherical aberration (SA), vertical coma (Vcoma), higher order root mean square (hRMS) and total RMS) were evaluated.

First AS-OCT and corneal topography measurements were performed. Then the Schirmer test, CFSS and TBUT were measured, in that order. The subjective discomfort related to dry eye was also assessed with the Turkish version of the Ocular Surface Disease Index (OSDI, Allergan Inc) questionnaire.

The statistical software package SPSS 18.0 (SPSS Inc., Chicago, Illinois, USA) for windows was used for data analysis. Data distribution for normality was assessed using Shapiro-Wilk test. Paired t-test or Wilcoxon test was used to determine the differences between the measurements before and 3 weeks after Btx injection. Data were presented as mean \pm standard deviations. A $P$ value of $<0.05$ was considered statistically significant.

\section{Results}

Thirty two eyes of 32 patients (17 female, 15 male) were included in the study. The mean age of the patients was $59.3 \pm 8.9$ years (39-71 years). The mean disease duration was $5.9 \pm 4$ years and the average time between the last and current BTX-A injection was $3.81 \pm 0.8$ months ( $3-6$ months).

The comparison of tear and ocular surface parameters at baseline and the 3rd week follow-up after BTXA injection are given in detail in Table 1. Tear meniscus height $(265.9 \pm 116.4 \mu \mathrm{m}$ vs. $347.1 \pm 172.6 \mu \mathrm{m} ; p$ $<0.001)$ and tear meniscus depth $(178.1 \pm 57.7 \mu \mathrm{m}$ vs. $221.4 \pm 90.3 \mu \mathrm{m} ; p<0.001)$ were significantly higher at the 3rd week compared to baseline (Fig. 1). There were no significant changes in TBUT, CFSS or the Schirmer I test after treatment. The OSDI score wassignificantly lower at the 3rd week after injection (27.8 \pm 26.1 to $18.4 \pm 19.8 p=0.04)$ (Fig. 2). 
Table 1

Changes in the tear and ocular surface parameters after BTX-A injection in patients with blepharospasm and hemifacial spasm

\begin{tabular}{|llll|}
\hline & $\begin{array}{l}\text { Before Injection } \\
\text { (n:32) }\end{array}$ & $\begin{array}{l}\text { 3 weeks after injection } \\
\text { (n:32) }\end{array}$ & p \\
\hline Tear meniscus height (mm) & $265,9 \pm 116,4$ & $347,1 \pm 172,6$ & $<0,001$ \\
\hline Tear meniscus depth (mm) & $178,1 \pm 57,7$ & $221,4 \pm 90,3$ & $<0,001$ \\
\hline Tear break-up time (sec) & $5,5 \pm 4,1$ & $6,15 \pm 4,2$ & 0,571 \\
\hline Corneal fluorescein staining & $1,9 \pm 2,1$ & $2,4 \pm 2,5$ & 0,168 \\
\hline Schirmer test (mm) & $7,6 \pm 5,5$ & $7,4 \pm 4,9$ & 0,640 \\
\hline OSDI score & $27,8 \pm 26,1$ & $18,4 \pm 19,8$ & $\mathbf{0 , 0 4}$ \\
\hline
\end{tabular}

K2 (44.1 $\pm 1.7 \mathrm{D}$ vs. $43.9 \pm 1.7 \mathrm{D} p=0.009)$ and mean $\mathrm{K}(43.7 \pm 1.6 \mathrm{D}$ vs. $43.6 \pm 1.6 \mathrm{D} p=0.025)$ and corneal astigmatism $(0.9 \pm 0.6 \mathrm{D}$ vs. $0.7 \pm 0.5 \mathrm{D} ; p=0.02)$, decreased significantly 3 weeks after the injection. The changes in the pachymetry and corneal aberrations were not statistically significant (Table 2, Fig. 3).

Table 2

Changes in corneal topography and corneal aberrations after BTX-A injection in patients with blepharospasm and hemifacial spasm

\begin{tabular}{|llll|}
\hline & $\begin{array}{l}\text { Before Injection } \\
(\mathbf{n}=\mathbf{3 2})\end{array}$ & $\begin{array}{l}\text { 3 weeks after injection } \\
(\mathbf{n}=\mathbf{3 2})\end{array}$ & $\mathbf{p}$ \\
\hline K1 & $43,2 \pm 1,6$ & $43,1 \pm 1,5$ & 0,48 \\
\hline K2 & $44,1 \pm 1,7$ & $43,9 \pm 1,7$ & $\mathbf{0 , 0 0 9}$ \\
\hline Kmean & $43,7 \pm 1,6$ & $43,6 \pm 1,6$ & $\mathbf{0 , 0 2 5}$ \\
\hline Thinnest corneal & $537,8 \pm 39,8$ & $544,1 \pm 27,9$ & 0,236 \\
thickness $(\boldsymbol{\mu})$ & & & \\
\hline Astigmatism & $0,9 \pm 0,6$ & $0,7 \pm 0,5$ & $\mathbf{0 , 0 2}$ \\
\hline Spherical aberration & $0,38 \pm 0,1$ & $0,4 \pm 0,1$ & 0,12 \\
\hline Vertical coma & $0,068 \pm 0,2$ & $0,07 \pm 0,2$ & 0,77 \\
\hline Horizontal coma & $-0,02 \pm 0,1$ & $-0,02 \pm 0,1$ & 0,61 \\
\hline Higher order RMS & $0,7 \pm 0,2$ & $0,6 \pm 0,2$ & 0,53 \\
\hline Total RMS & $2,6 \pm 1,1$ & $2,4 \pm 1,1$ & 0,27 \\
\hline
\end{tabular}




\section{Discussion}

BTX-A has been used to treat HFS and BEB since the 1980s.[19] It is a safe and effective treatment that relieves the symptoms of BEB and HFS in $66-100 \%$ of cases $[20,21]$.

The results of studies showing the relationship between dry eye and BTX-A treatment are contradictory. Although evaluation times are different, at the time when the effect of BTX-A is maximum, the TBUT and Schirmer test results are different between studies. While a study reported an increase in TBUT and Schirmer test [4], Kocabeyoglu et al. reported an increase in TBUT, no change in Schirmer test [22], others have shown an increase in TBUT and decrease in Schirmer test $[9,23]$. The most likely cause of these different results is the differences in injection sites and dosages.

BTX-A injection into the lacrimal gland reduces aqueous tear secretion so it is used in the treatment of epiphora. Conversely, BTX-A injection to the lower medial canthal region is used in dry eye treatment. It decreases the blinking rate, reduces the action of the lacrimal pump and increases tear retention due to paralysis of the orbicularis muscle $[24,25]$.

Lu et al. showed that inflammation related cytokines, like TNF-a, IL-6 and IL-1ß, are higher in patients with BEB and DED compared to patients with only DED. The authors concluded that blinking in BEB patients leads to micro abrasive effects in the conjunctiva and increases inflammation [5]. Ho et al. showed that lipid tear thickness is significantly increased, without any change in meibomian gland dropout, when compared to baseline at the first month of BTX-A treatment. They concluded that this effect is due to paralysis of the lacrimal pump and the retention of tears on the ocular surface [26].

According to the TFOS DEWS II report, tear film thickness (TFT) is one of the dry eye sub-classification methods, and the tear film is composed of two layers; the muco-aqueous gel layer underneath, and an overlying lipid layer [27]. OCT measures the height, area and curvature of the upper and lower menisci and, although these parameters don't show the central tear film, the lower tear meniscus height shows the muco-aqueous tear volume $[12,28,29]$. In this study, after BTX-A treatment, TMH and TMD were significantly increased and OSDI scores, which evaluate the patients' symptoms, decreased significantly. TMH and TMD correlate well with dry eye symptoms [30]. Gunes et al. and Lu et al. also reported a significant decrease in OSDI scores after BTX-A injections in BEB and HFS patients [5, 9]. Despite the improvement in OSDI scores, there were no significant changes in TBUT, Schirmer I or CFSS. This finding can be related to the increase in inflammatory mediators on the ocular surface and can be explained by the poor correlation between dry eye symptoms and signs including via the Schirmer test and ocular staining, as reported previously [31].

In the present study, TMH and TMA increased significantly after BTX-A injection. In a recent study, Park et al. evaluated lacrimal drainage and TMH in BEB patients and showed an increase in $\mathrm{TMH}$ and tear 
clearance time after BTX-A treatment [4]. The results of these studies suggest that BTX-A related paralysis of the orbicularis muscle leads to a decrease in nasolacrimal outflow and tear retention.

Eyelid pressure is an important cause of corneal astigmatism. Previous studies have reported corneal topographic changes after ptosis, blepharoplasty and gold-weight implant surgery [32, 33]. Gunes et al. evaluated corneal topographic changes after BTX-A treatment in HFS and BEB patients and reported a significant decrease in astigmatism [9]. Similarly, Osaki et al. reported that steep keratometry and astigmatism decreased significantly at 2 months and 3 months after treatment in HFS patients [34]. While the decrement in steep $\mathrm{K}$, mean $\mathrm{K}$ and astigmatism values was statistically significant 3 weeks after BTX-A injections, the change between the baseline and 3rd week measurements ( $0.2 \mathrm{D}$ for the steep $\mathrm{K}$ and $0.1 \mathrm{D}$ for the mean $\mathrm{K}$ ) was very close to the repeatability limits of Pentacam [35].

In the present study, the changes in anterior corneal aberrations after BTX-A treatment were also investigated. Previous studies have shown that abnormalities in the tear film and an increase in the interblink interval increase corneal and total aberrations and effect visual quality [36-39]. Isshiki et al. evaluated total ocular aberration and reported a significant decrease in HOA in BEB patients after relieving spasms [16]. Yakibu et al. reported a significant decrease in total hRMS and SA after treatment in BEB and HFS patients [17]. This is the first study to evaluate anterior corneal aberrations after BTX-A injections in patients with BEB and HFS. Although hRMS and tRMS decreased after treatment, the results were not significant). The difference between these results may be explained by the difference in the measured aberrations, that is, ocular versus corneal. An improvement in tear functions and decreased blinking rate can change the quality of light transmitted to intraocular structures which causes an improvement in corneal and other ocular aberrations.

There are some limitations of this study. Firstly, the number of participants is low and, secondly, all patients included in the study had undergone long-term BTX-A treatment. As the effect of BTX-A can last for up to 6 months, the previous BTX-A effect may not be completely over at the time participants were enrolled for the study. Finally, the Schirmer test was completed first so this might have affected the CFSS results.

In conclusion, BTX-A injection increases tear meniscus and decrease symptoms related to dry eye disease in BEB and HFS patients. It decrease astigmatism and keratometry values but it does not cause a significant change in corneal aberrations.

\section{Declarations}

Conflict of Interest The authors declare that they have no conflict of interest.

\section{Funding None.}


Author Contributions Dr. Bayraktar Bilen - Data collection, manuscript submission and coordination, statistical analysis; Dr. Bilen - Coordination and data collection; Dr. Topçu Yılmaz - Data and Manuscript Editing; and Evren Kemer - Consultants to the procedure.

Data availability The manuscript has no associated data in a data repository.

Animal Research This article does not contain any studies with animals performed by any of the authors.

Consent to Participate Informed consent was obtained from all individual participants included in the study.

Consent to Publish Informed consent for potential research publication was obtained from all individual participants included in the study at the time of their treatments performed.

Ethical approval All procedures performed in studies involving human participants were in accordance with the ethical standards of the institutional and/or national research committee (Ankara Numune Education and Research Hospital E-19-2606) and with the 1964 Helsinki declaration and its later amendments or comparable ethical standards.

\section{References}

1. Anderson RL, Patel B, Holds JB, Jordan DR (1998) Blepharospasm: past, present, and future. Ophthalmic plastic and reconstructive surgery 14 (5):305-317

2. Chaudhry N, Srivastava A, Joshi L (2015) Hemifacial spasm: the past, present and future. Journal of the neurological sciences 356 (1-2):27-31

3. Osako M, Keltner JL (1991) Botulinum A toxin (Oculinum $\left.{ }^{\circledR}\right)$ in ophthalmology. Survey of ophthalmology 36 (1):28-46

4. Park DI, Shin HM, Lee SY, Lew H (2013) Tear production and drainage after botulinum toxin A injection in patients with essential blepharospasm. Acta ophthalmologica 91 (2):e108-e112

5. Lu R, Huang R, Li K, Zhang X, Yang H, Quan Y, Li Q (2014) The influence of benign essential blepharospasm on dry eye disease and ocular inflammation. American journal of ophthalmology 157 (3):591-597 e591-592. doi:10.1016/j.ajo.2013.11.014

6. Craig JP, Nichols KK, Akpek EK, Caffery B, Dua HS, Joo C-K, Liu Z, Nelson JD, Nichols JJ, Tsubota K (2017) TFOS DEWS II definition and classification report. The ocular surface 15 (3):276-283

7. Ho MC, Hsu WC, Hsieh YT (2014) Botulinum toxin type a injection for lateral canthal rhytids : effect on tear film stability and tear production. JAMA ophthalmology 132 (3):332-337.

doi:10.1001/jamaophthalmol.2013.6243

8. Bilyk JR, Yen MT, Bradley EA, Wladis EJ, Mawn LA (2018) Chemodenervation for the Treatment of Facial Dystonia: A Report by the American Academy of Ophthalmology. Ophthalmology 125 (9):1459-1467 
9. Gunes A, Demirci S, Koyuncuoglu HR, Tok L, Tok O (2015) Corneal and Tear Film Changes After Botulinum Toxin-A in Blepharospasm or Hemifacial Spasm. Cornea 34 (8):906-910. doi:10.1097/ICO.0000000000000482

10. Palakuru JR, Wang J, Aquavella JV (2007) Effect of blinking on tear dynamics. Investigative ophthalmology \& visual science 48 (7):3032-3037

11. Ang M, Baskaran M, Werkmeister RM, Chua J, Schmidl D, dos Santos VA, Garhoefer G, Mehta JS, Schmetterer $L$ (2018) Anterior segment optical coherence tomography. Progress in retinal and eye research 66:132-156

12. Shen M, Li J, Wang J, Ma H, Cai C, Tao A, Yuan Y, Lu F (2009) Upper and lower tear menisci in the diagnosis of dry eye. Investigative ophthalmology \& visual science 50 (6):2722-2726

13. Nguyen P, Huang D, Li Y, Sadda SR, Ramos S, Pappuru RR, Yiu SC (2012) Correlation between optical coherence tomography-derived assessments of lower tear meniscus parameters and clinical features of dry eye disease. Cornea 31 (6):680

14. Moon NJ, Lee HI, Kim JC (2006) The changes in corneal astigmatism after botulinum toxin-a injection in patients with blepharospasm. Journal of Korean medical science 21 (1):131-135

15. Koh S (2018) Irregular astigmatism and higher-order aberrations in eyes with dry eye disease. Investigative ophthalmology \& visual science 59 (14):DES36-DES40

16. Isshiki Y, Ishikawa H, Mimura $O$ (2016) Changes in ocular higher-order aberrations following botulinum toxin treatment in patients with blepharospasm. Japanese journal of ophthalmology 60 (6):486-491

17. Yabiku MM, Sartori FJ, Sarraff EP, Osaki TH, Hossaka SK, Pereira Cl, Freitas W, Osaki MH, Cariello AJ (2011) Ocular wavefront aberrations in patients with facial dystonia treated with botulinum toxin. Arquivos brasileiros de oftalmologia 74 (6):414-416

18. Denoyer A, Rabut G, Baudouin C (2012) Tear film aberration dynamics and vision-related quality of life in patients with dry eye disease. Ophthalmology 119 (9):1811-1818

19. Mauriello Jr JA, Coniaris H, Haupt EJ (1987) Use of Botulinum Toxin in the Treatment of One Hundred Patients with Facial Dyskinesias. Ophthalmology 94 (8):976-979

20. Jost WH, Kohl A (2001) Botulinum toxin: evidence-based medicine criteria in blepharospasm and hemifacial spasm. Journal of neurology 248 (1):I21-I24

21. Defazio G, Abbruzzese G, Girlanda P, Vacca L, Curra A, De Salvia R, Marchese R, Raineri R, Roselli F, Livrea P (2002) Botulinum toxin A treatment for primary hemifacial spasm: a 10-year multicenter study. Archives of Neurology 59 (3):418-420

22. Kocabeyoglu S, Sekeroglu HT, Mocan MC, Muz E, Irkec M, Sanac AS (2014) Ocular surface alterations in blepharospasm patients treated with botulinum toxin A injection. European journal of ophthalmology 24 (6):830-834

23. Horwath-Winter J, Bergloeff J, Floegel I, Haller-Schober E, Schmut O (2003) Botulinum toxin A treatment in patients suffering from blepharospasm and dry eye. British journal of ophthalmology 87 (1):54-56 
24. Ziahosseini K, Al-Abbadi Z, Malhotra R (2015) Botulinum toxin injection for the treatment of epiphora in lacrimal outflow obstruction. Eye 29 (5):656

25. Sahlin S, Linderoth R (2008) Eyelid botulinum toxin injections for the dry eye. In: Surgery for the Dry Eye, vol 41. Karger Publishers, pp 187-192

26. Ho RW, Fang PC, Chao TL, Chien CC, Kuo MT (2018) Increase lipid tear thickness after botulinum neurotoxin A injection in patients with blepharospasm and hemifacial spasm. Scientific reports 8 (1):8367. doi:10.1038/s41598-018-26750-5

27. Willcox MD, Argüeso P, Georgiev GA, Holopainen JM, Laurie GW, Millar TJ, Papas EB, Rolland JP, Schmidt TA, Stahl U (2017) TFOS DEWS II tear film report. The ocular surface 15 (3):366-403

28. Wang J, Aquavella J, Palakuru J, Chung S, Feng C (2006) Relationships between central tear film thickness and tear menisci of the upper and lower eyelids. Investigative ophthalmology \& visual science 47 (10):4349-4355

29. Savini G, Barboni P, Zanini M (2006) Tear meniscus evaluation by optical coherence tomography. Ophthalmic Surgery, Lasers and Imaging Retina 37 (2):112-118

30. Demirok GS, Gurdal C, Sarac O, Ceran BB, Can I (2013) Evaluating of tear meniscus parameters with optical coherent tomography in dry-eye patients/Kuru Goz Hastalarinda Optik Koherans Tomografi ile Gozyasi Meniskus Parametrelerinin Degerlendirilmesi. Turkish journal of ophthalmology 43 (4):258-263

31. Schein OD, Tielsch JM, Muñoz B, Bandeen-Roche K, West S (1997) Relation between signs and symptoms of dry eye in the elderly: a population-based perspective. Ophthalmology 104 (9):13951401

32. Kim YK, In JH, Jang SY (2016) Changes in Corneal Curvature After Upper Eyelid Surgery Measured by Corneal Topography. Journal of Craniofacial Surgery 27 (3):e235-e238

33. Mavrikakis I, Beckingsale P, Lee E, Riaz Y, Brittain P (2006) Changes in corneal topography with upper eyelid gold weight implants. Ophthalmic Plastic \& Reconstructive Surgery 22 (5):331-334

34. Osaki T, Osaki MH, Osaki TH, Hirai FE, Campos M (2016) Differences in Corneal Parameters Between Affected and Normal Contralateral Eyes in Patients With Hemifacial Spasm Treated With Botulinum Toxin-A: Outcomes During One Complete Treatment Cycle. Cornea 35 (2):220-225

35. McAlinden C, Khadka J, Pesudovs K (2011) A comprehensive evaluation of the precision (repeatability and reproducibility) of the Oculus Pentacam HR. Investigative ophthalmology \& visual science 52 (10):7731-7737. doi:10.1167/iovs.10-7093

36. Rieger $\mathrm{G}$ (1992) The importance of the precorneal tear film for the quality of optical imaging. British journal of ophthalmology 76 (3):157-158

37. Koh S, Maeda N, Kuroda T, Hori Y, Watanabe H, Fujikado T, Tano Y, Hirohara Y, Mihashi T (2002) Effect of tear film break-up on higher-order aberrations measured with wavefront sensor. American journal of ophthalmology 134 (1):115-117

38. Montés-Micó R, Alió JL, Munoz G, Charman WN (2004) Temporal changes in optical quality of airtear film interface at anterior cornea after blink. Investigative ophthalmology \& visual science 45 
(6):1752-1757

39. Montés-Micó R, Alió JL, Muñoz G, Pérez-Santonja JJ, Charman WN (2004) Postblink changes in total and corneal ocular aberrations. Ophthalmology 111 (4):758-767

Figures

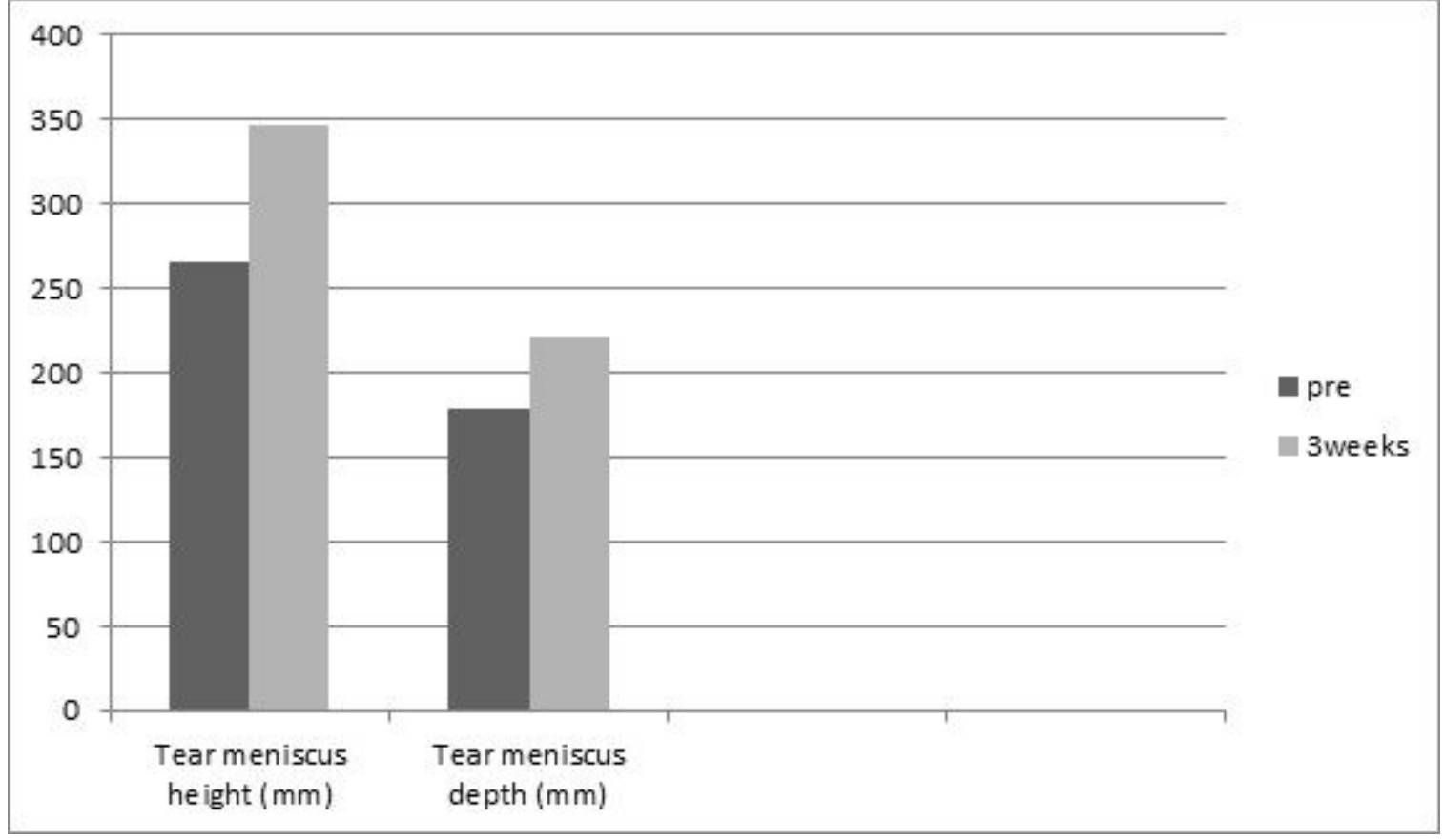

\section{Figure 1}

Comparison of tear meniscus height and tear meniscus dept before and 3 weeks after BTX-A injection

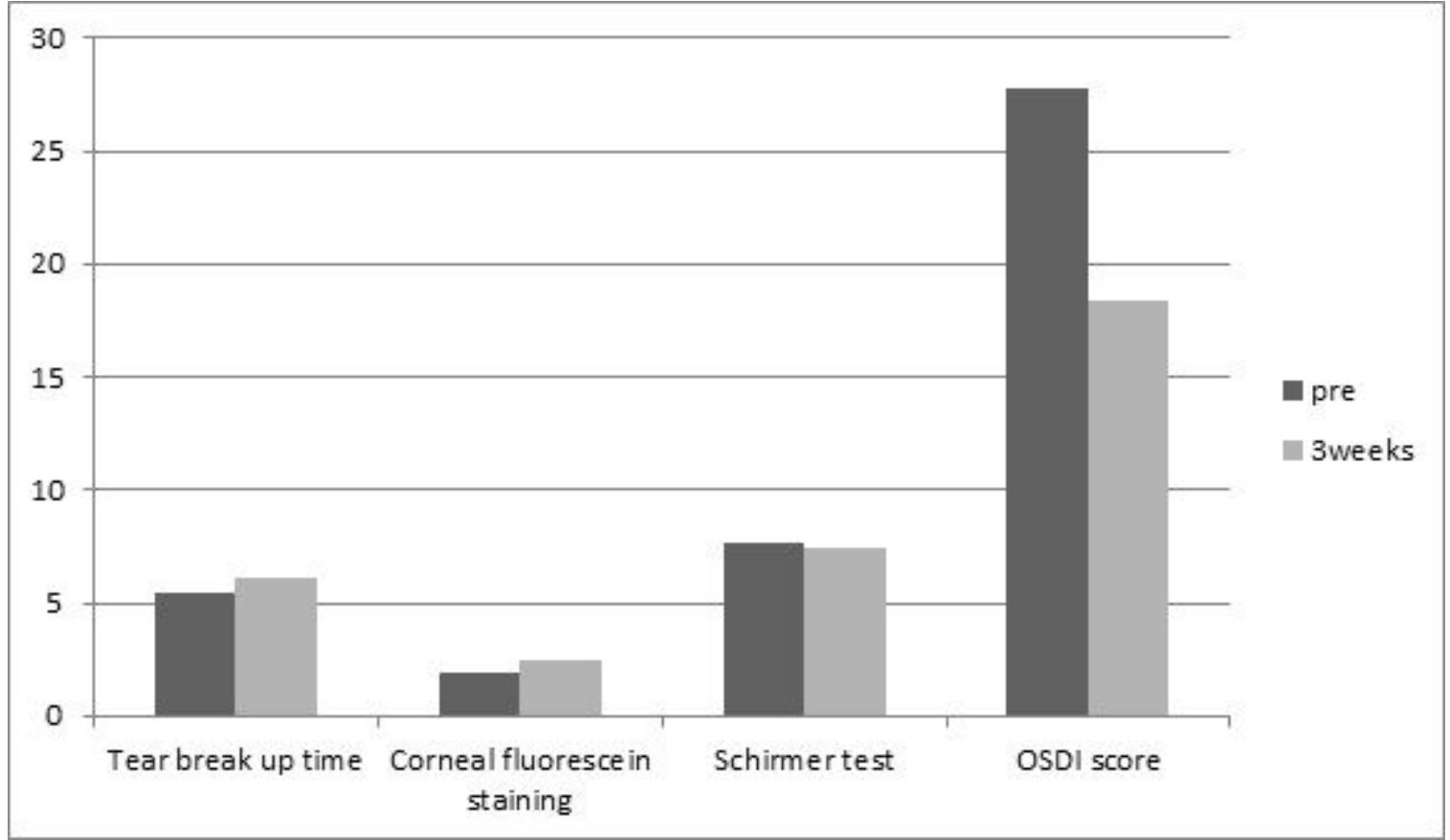

Figure 2 
Comparison of the dry eye tests and OSDI score before and 3 weeks after BTX-A injection

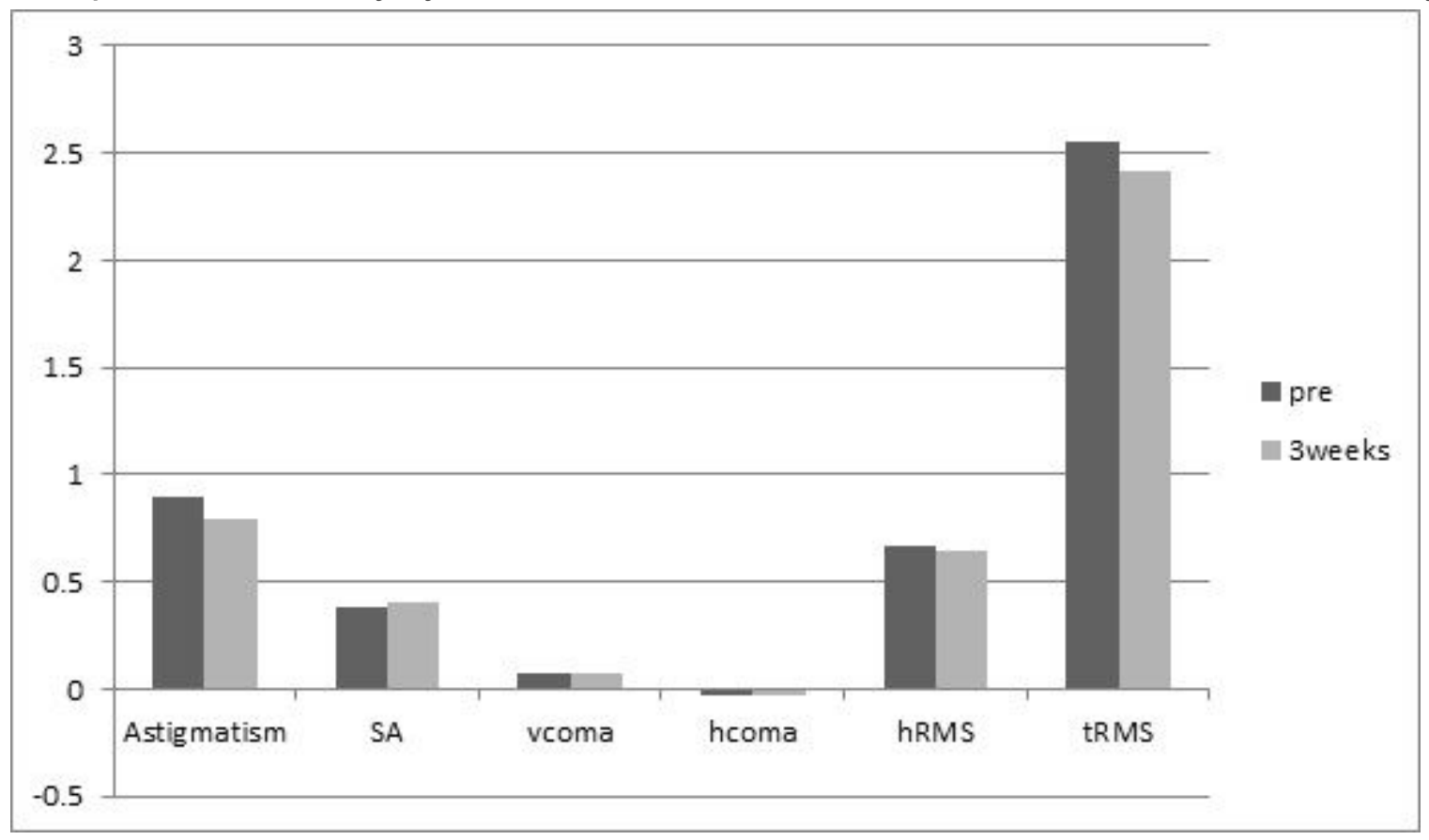

\section{Figure 3}

Comparison of astigmatism and corneal aberrations before and 3 weeks after BTX-A injection. (SA: spheric aberration; vcoma: vertical coma; hcoma: horizontal coma; hRMS: higher order root mean square; tRMS: total root mean square) 